\title{
Laparoscopic Cholecystostomy in Gangrenous Cholecystitis with Localized Peritonitis
}

\author{
P Balaji ${ }^{1}$, Abhinav Balaji ${ }^{2}$, RV Ramanakumar ${ }^{3}$, Midhun M John $^{4}$
}

\begin{abstract}
Introduction: Gallstone is a very common disease condition and affects $10-20 \%$ of the adults in the developed countries and $20 \%$ of the patients present with acute calculous cholecystitis. ${ }^{1}$ One of the severe complications is gangrenous cholecystitis, which can occur in as high as $40 \%{ }^{2,3}$ of patients with acute cholecystitis, and perforation of gallbladder (GB) in $2-18 \%{ }^{4}$ Gangrenous cholecystitis is defined as necrosis and perforation of the GB wall as a result of ischemia following progressive vascular insufficiency and is a severe complication of cholelithiasis. Factors such as male sex, advanced age, delay in seeking treatment, leukocytosis, cardiovascular diseases, and diabetes mellitus increase the likelihood of developing gangrenous cholecystitis and carry a significantly higher mortality rate between $15 \%$ and $50 \% .{ }^{5} \mathrm{Hence}$, early diagnosis and immediate intervention are required in these cases. Laparoscopic cholecystectomy for gangrenous cholecystitis carries a high risk of morbidity and mortality. Hence, safer treatment modalities such as laparoscopic cholecystostomy help the patient to recover from the critical illness and the definitive procedure can be performed at a later, safer period.

Case description: Six patients with gangrenous cholecystitis, i.e., five females and one male, underwent laparoscopic cholecystostomy. All the patients recovered from sepsis, and no complication was reported during or after the procedure and were discharged after a stay of 5-7 days. All the patients underwent elective laparoscopic cholecystectomy after 10-12 weeks and are doing well at 1-year follow-up.

Conclusion: Cholecystectomy in gangrenous cholecystitis carries high risk of morbidity and mortality. In this setting, laparoscopic cholecystostomy is a safe and reliable procedure to recover the patient from the acute sepsis and proceed with elective laparoscopic cholecystectomy at a later date. Keywords: Gangrenous cholecystitis, Interval cholecystectomy, Laparoscopic cholecystostomy.

The Journal of Medical Sciences (2019): 10.5005/jp-journals-10045-00128
\end{abstract}

\section{BACKGROUND}

Gallstone is a very common disease condition and can affect 10 to $20 \%$ of the adults in the developed countries, up to $20 \%$ patients present with acute calculous cholecystitis. ${ }^{1}$ One of the severe complications is gangrenous cholecystitis and can occur in as high as $40 \%{ }^{2,3}$ patients with acute cholecystitis and perforation of GB in $2-18 \% .{ }^{4}$ Morbidity and mortality rates are high with gangrenous cholecystitis and demonstrated the mortality rate of as high as $50 \%,{ }^{5}$ so early diagnosis and immediate interventions are required in these cases.

We report a case series of five patients who presented with features of gangrenous calculous cholecystitis and who underwent laparoscopic cholecystostomy.

\section{Case Description}

A 60-year-old hypothyroid female patient got admitted with gangrenous cholecystitis after being treated for 15 days at a local hospital conservatively for acute calculus cholecystitis. On admission, she had rebound tenderness and a palpable mass size of $10 \mathrm{~cm}$ in right hypochondrium and lumbar region. Computed tomography (CT) scan with intravenous contrast revealed distended GB with pericholecystic fat stranding. A defect was seen in the lateral aspect of the fundus which communicates to a collection that tracts through the right side of the liver up to the dome of the diaphragm (Fig. 1) with right pleural effusion. Common bile duct showed sludge (Fig. 2).

She was started on broad-spectrum antibiotics empirically and intravenous fluids and underwent endoscopic retrograde cholangiopancreatography (ERCP) and was found to have parapapillary choledochodudenal fistula for which fistulotomy was done and stent was placed in the common bile duct (CBD). Percutaneous drainage was attempted but was not successful because of the thick nature \begin{tabular}{l}
\hline${ }^{1-4}$ Department of General Surgery, Apollo Hospitals, Chennai, Tamil \\
Nadu, India \\
Corresponding Author: Abhinav Balaji, Department of General
\end{tabular} Surgery, Apollo Hospitals, Chennai, Tamil Nadu, India, Phone: +91 9551291515, e-mail: abhinavbalaji15@gmail.com

How to cite this article: Balaji P, Balaji A, Ramanakumar RV, et al. Laparoscopic Cholecystostomy in Gangrenous Cholecystitis with Localized Peritonitis. J Med Sci 2019;5(3):77-79.

Source of support: Nil

Conflict of interest: None

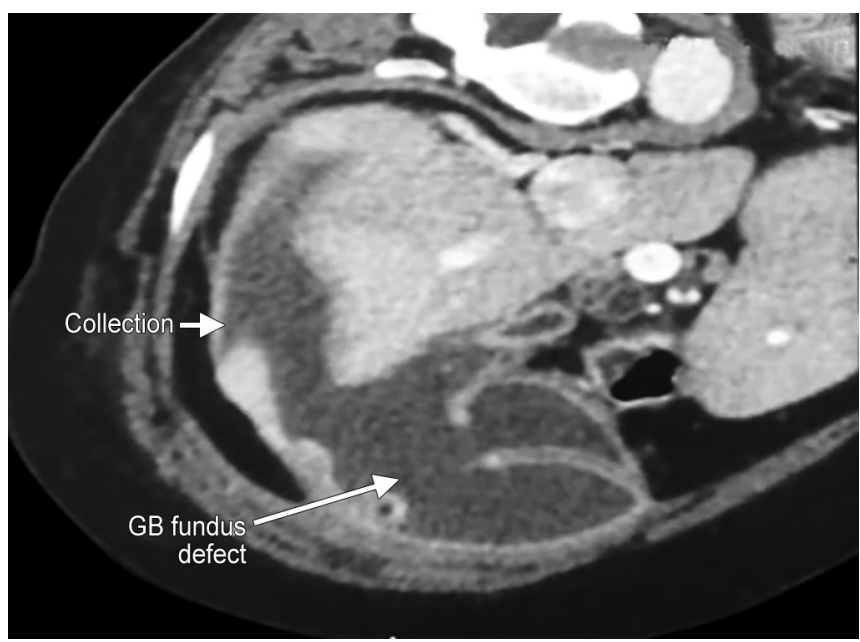

Fig. 1: Computed tomography scan of gallbladder showing funda defect and collection in the right subdiaphragmatic space extending up to the dome of diaphragm

o The Author(s). 2019 Open Access This article is distributed under the terms of the Creative Commons Attribution 4.0 International License (https://creativecommons. org/licenses/by-nc/4.0/), which permits unrestricted use, distribution, and non-commercial reproduction in any medium, provided you give appropriate credit to the original author(s) and the source, provide a link to the Creative Commons license, and indicate if changes were made. The Creative Commons Public Domain Dedication waiver (http://creativecommons.org/publicdomain/zero/1.0/) applies to the data made available in this article, unless otherwise stated. 


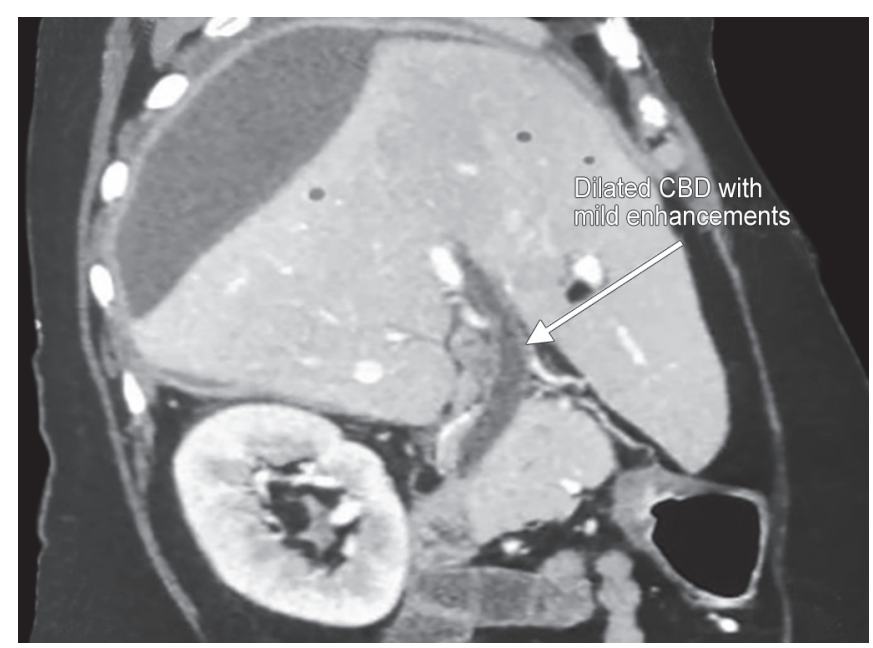

Fig. 2: Computed tomography scan showing CBD with mild enhancement

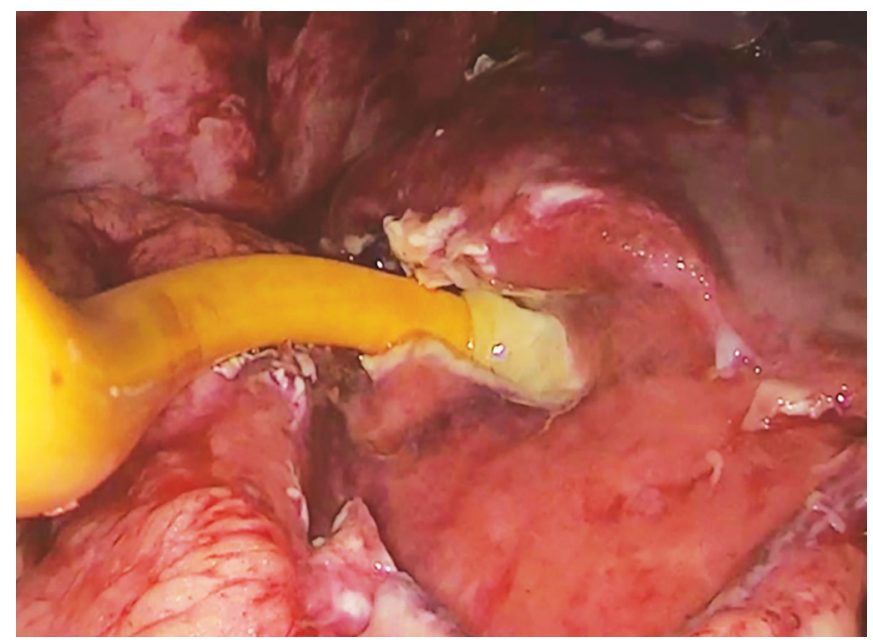

Fig. 4: Foley's tube being inserted into the gallbladder through the fundal perforation

of the pus and hence was taken up for diagnostic laparoscopy and proceeded. At laparoscopy, falciform ligament, transverse colon, and duodenum all adhered to the undersurface of liver and to the anterior abdominal wall, and the liver was plastered to the undersurface of the diaphragm and rib cage on the right side.

Liver was released from the undersurface of the right rib cage. After the anterior abdominal wall adhesions were released by blunt dissection, pus collection was observed around the fundus which was walled by bowel and anterior abdominal wall let out, and the small bowel and omentum separated from the undersurface of the liver. A defect of $1 \mathrm{~cm}$ was seen in the fundus of $G B$ with gangrenous wall, and the rest of the GB was not delineated. There was another abscess cavity in the right subdiaphragmatic space extending up to the dome of the diaphragm and deroofing done and about $150 \mathrm{~mL}$ pus was drained and send for culture and sensitivity (Fig. 3).

Multiple pearly white stones were removed from the GB. After a thorough wash, $32 \mathrm{~F}$ chest drain was placed in the right subdiaphragmatic space, 20F Foley's tube was inserted by transperitoneal technique into the GB (Fig. 4) and the balloon was inflated using $20 \mathrm{~mL}$ sterile water.

She was shifted to surgical high-dependency unit postoperatively and recovered well, was discharged with subhepatic drain, and underwent laparoscopic cholecystectomy after 3 months.

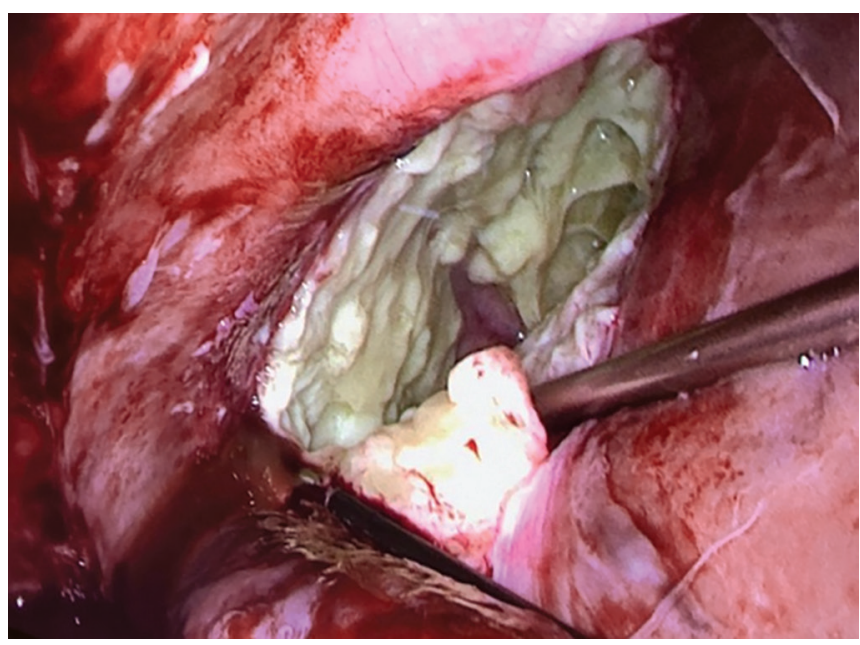

Fig. 3: Deroofing of abscess cavity in the right subdiaphragmatic space

Another five similar cases of gangrenous cholecystitis presented with upper abdominal pain for 5-10 days' duration, and ultrasound showed features of acute cholecystitis and CT scan revealed GB perforation with features of gangrenous cholecystitis. All the patients were treated with laparoscopic cholecystostomy and recovered well and underwent elective interval cholecystectomy after 2-3 months. All patients are doing well at 1-year follow-up.

\section{Discussion}

Even though the incidence is low, acute cholecystitis can cause GB perforation in $2-18 \%{ }^{4}$ of patients and most common location was found to be in the fundus of the GB. Niemeier et al. ${ }^{6}$ classified GB perforation into three types, i.e.,

Type I (acute): free perforation with generalized peritonitis.

Type II (subacute): localized peritonitis.

Type III (chronic): cholecystoenteric fistula of the GB with or without cholelithiasis.

In our study, five of our patients were female and one was male; and four of them were aged between 55 and 65, the oldest patient being 64 years. One patient was 48 -year-old. The study patients' average age being 58 years.

Acute uncomplicated cholecystitis is more common among females, with a female to male ratio of 2:1. ${ }^{7}$ However, GB perforation was more frequent in male gender. ${ }^{4,8}$ Our study consisted of more females, with a male to female ratio of 1:5 (Table 1).

All patients had an elevated white blood cell (WBC) count, with an average value of 14,222 . Parker et al., ${ }^{9}$ increased WBC count are not the diagnostic indications for GB perforation. Roslyn and Busuttil ${ }^{4}$ reported that type I and type II GB perforation mostly occur in younger patients, around the age of 50 years, whereas type III GB perforation is more common in the elderly individuals. Our study being a small series, no significant findings were observed with age and type of perforation.

In our case series, the CT showed GB perforation in five patients and all of them were in the fundal region, thus diagnosis of GB perforation were made preoperatively. According to Tanaka et al.'s study, ${ }^{10}$ only one out of nine patients had a concrete diagnosis preoperatively. In majority of the patients, the GB perforation was identified intraoperatively and these patients underwent surgery with a preoperative diagnosis of acute cholecystitis. 
Laparoscopic Cholecystostomy in Gangrenous Cholecystitis with Localized Peritonitis

Table 1: Patient variables and number count

\begin{tabular}{ll}
\hline Patients' variables & \\
\hline Male to female ratio & $1: 5$ \\
Average age & 58 years \\
Diabetes mellitus $(n)$ & 5 \\
Known case of gallstone disease $(n)$ & 5 \\
Elevated WBC count $(n)$ & 6 \\
Average duration of symptoms & 7 days \\
Type I perforation $(n)$ & 2 \\
Type II perforation $(n)$ & 3 \\
Type III perforation $(n)$ & 0 \\
Empyema GB & 1 \\
\hline
\end{tabular}

$n$, number of patients

For the diagnosis of GB perforation, the $\mathrm{CT}$ scan is ideal. Ultrasound findings in acute cholecystitis, such as the GB wall thickening, GB distension, pericholecystic free fluid, and positive sonographic Murphy sign, may also be present in GB perforation cases. ${ }^{10-12}$ On the contrary, CT scan accurately picks up signs of free intraperitoneal fluid, pericholecystic fluid, GB wall thickness, and the defect on the wall due to perforation. , $^{10,14}$

The mortality rate is high in patients with GB perforation. Hence, early diagnosis, stabilization of patient, and intervention are essential. Cholecystectomy, drainage of abscess, and abdominal lavage are the necessary treatment of GB perforation. ${ }^{4,15}$ The reported mortality rate is up to $7 \%$ in emergency cholecystectomy. ${ }^{16}$ Cholecystostomy is a reliable option for patients with biliary sepsis and can be done by ultrasound guidance or laparoscopically.

In our case series, we did laparoscopic cholecystostomy for all the six patients, i.e., five of them with perforation and one having empyema GB with dense adhesions. Cholecystostomy ensues external drainage of the infected GB through a tube inserted into it, which can be achieved by percutaneous method under ultrasound guidance, open method, and laparoscopic approach. Of these, laparoscopic cholecystostomy is considered the best, as the surgeon can assess the disease severity and avoid trauma to the hepatic flexure and duodenum. ${ }^{17,18}$ All the six patients underwent ERCP and CBD stenting prior to laparoscopic cholecystostomy. One patient had incidental finding of choledochoduodenal fistula, which was disconnected during the ERCP procedure.

Laparoscopic cholecystostomy can be done in two ways, namely, transperitoneal and transcannular techniques. In transperitoneal technique, the Foley's catheter is introduced into the perforated site of the GB and the balloon is inflated and finally fixed to the anterior abdominal wall. In transcannular technique, the trocar is used to puncture the GB, the catheter is introduced through the cannula into the $\mathrm{GB}$, and the balloon is inflated. ${ }^{16}$ Laparoscopic cholecystostomy is a safe procedure with minimum complications, including dislodgment of Foley's catheter, bile leakage into the intraperitoneal cavity, and bleeding. ${ }^{17}$ So in a critically ill patient, resolution of sepsis can be achieved by cholecystostomy; and a definitive procedure like cholecystectomy can be planned electively when the patient is stable in calculous cholecystitis. No further procedure is required in case of acalculous cholecystitis. In our series, the average hospital stay for patients with GB perforation was 13 days. In a study by Nandyala et al., ${ }^{19}$ it was 8 days and 15 days for calculous and acalculous cholecystitis, respectively. In our series, all patients underwent interval cholecystectomy, five of them were managed laparoscopically and one was converted to open cholecystectomy due to unclear anatomy. Average duration from laparoscopic cholecystostomy to interval cholecystectomy was 82 days.

\section{Conclusion}

Cholecystostomy is an ideal procedure for patients in biliary sepsis associated with acute cholecystitis and can reduce the surrounding inflammation by definite elective cholecystectomy. Early diagnosis and emergency surgery are crucial in surgical management of gangrenous cholecystitis because of the high morbidity and mortality. So from our experience, we can conclude that laparoscopic cholecystostomy is a safe procedure and should be offered to patients who have GB perforation especially in patients with comorbid conditions and who are critically ill.

\section{References}

1. Indar AA, Beckingham IJ. Acute cholecystitis. BMJ 2002;325(7365): 639-643. DOI: 10.1136/bmj.325.7365.639.

2. Fagan SP, Awad SS, Rahwan K, et al. Prognostic factors for the development of gangrenous cholecystitis. Am J Surg 2003;186(5): 481-485. DOI: 10.1016/j.amjsurg.2003.08.001.

3. Aydin C, Altaca G, Berber I, et al. Prognostic parameters for the prediction of acute gangrenous cholecystitis. J Hepatobiliary Pancreat Surg 2006;13(2):155-159. DOI: 10.1007/s00534-005-1042-8.

4. Roslyn J, Busuttil RW. Perforation of the gallbladder: a frequently mismanaged condition. Am J Surg 1979;137(3):307-312. DOI: 10.1016/0002-9610(79)90056-4.

5. Habib FA, Kolachalam RB, Khilnani R, et al. Role of laparoscopic cholecystectomy in the management of gangrenous cholecystitis. Am J Surg 2001;181(1):71-75. DOI: 10.1016/S0002-9610(00)00525-0.

6. Niemeier OW. Acute free perforation of the gall-bladder. Ann Surg 1934;99(6):922. DOI: 10.1097/00000658-193499060-00005.

7. Glenn F. Acute cholecystitis. Surg Gynecol Obstet 1976;143(1):56-60.

8. Bedirli A, SakrakO, Sözüer EM, et al. Factors effecting the complications in the natural history of acute cholecystitis. Hepatogastroenterology 2001;48(41):1275-1278.

9. Parker LJ, Vukov LF, Wollan PC. Emergency department evaluation of geriatric patients with acute cholecystitis. Acad Emerg Med 1997;4(1):51-55. DOI: 10.1111/j.1553-2712.1997.tb03643.x.

10. Tanaka M, Takahashi H, Yajima Y, et al. Idiopathic perforation of the gallbladder: report of a case and a review of the Japanese literature. Surg Today 1997;27(4):360-363. DOI: 10.1007/BF00941814.

11. Sood BP, Kalra N, Gupta S, et al. Role of sonography in the diagnosis of gallbladder perforation. J Clin Ultrasound 2002;30(5):270-274. DOI: 10.1002/jcu.10071.

12. Menakuru SR, Kaman L, Behera A, et al. Current management of gallbladder perforations. ANZ J Surg 2004;74(10):843-846. DOI: 10.1111/j.1445-1433.2004.03186.x.

13. Wang AJ, Wang TE, Lin CC, et al. Clinical predictors of severe gallbladder complications in acute acalculous cholecystitis. World J Gastroenterology 2003;9(12):2821. DOI: 10.3748/wjg.v9.i12.2821.

14. Van Sonnenberg E, D'Agostino H, Casola G. Interventional gallbladder procedures. Radiol Clin North Am 1990;28(6):1185.

15. Ong CL, Wong $\mathrm{TH}$, Rauff A. Acute gall bladder perforation-a dilemma in early diagnosis. Gut 1991;32(8):956-958. DOI: 10.1136/gut.32.8.956.

16. Chowbey PK, Venkatasubramanian R, Bagchi N, et al. Laparoscopic cholecystostomy is a safe and effective alternative in critically ill patients with acute cholecystitis: two cases. J Laparoendosc Adv Surg Tech A 2007;17(1):43-46. DOI: 10.1089/lap.2006.05078.

17. Cuschieri A, Berci G. Laparoscopic cholecystolithotomy and cholecystostomy. In: Laparoscopic Biliary Surgery. Oxford: Blackwell Scientific; 1992. pp. 148-154.

18. Cuschieri A. Cholecystostomy drainage for severe acute cholecystitis. In: Operative Manual of Endoscopic Surgery. Berlin, Heidelberg: Springer; 1992. pp. 240-245.

19. Nandyala VN, Chintakindi SB, Pallagani L, et al. Gall bladder perforation-is it still a diagnostic dilemma: a retrospective study. Int Surg J 2016;3(2):609-613. DOI: 10.18203/2349-2902.isj20161131. 